\title{
Distinguishing primary adenocarcinoma of the urinary bladder from secondary involvement by colorectal adenocarcinoma: extended immunohistochemical profiles emphasizing
} novel markers

Qiu Rao ${ }^{1,2}$, Sean R Williamson ${ }^{1}$, Antonio Lopez-Beltran ${ }^{3}$, Rodolfo Montironi ${ }^{4}$, Wenbin Huang $^{1,5}$, John N Eble ${ }^{1}$, David J Grignon ${ }^{1}$, Michael O Koch ${ }^{6}$, Muhammad T Idrees ${ }^{1}$, Robert E Emerson $^{1}$, Xiao-Jun Zhou ${ }^{2}$, Shaobo Zhang ${ }^{1}$, Lee Ann Baldridge ${ }^{1}$ and Liang Cheng ${ }^{1,6}$

${ }^{1}$ Department of Pathology, Indiana University School of Medicine, Indianapolis, IN, USA; ${ }^{2}$ Department of Pathology, Nanjing Jinling Hospital, Nanjing University School of Medicine, Nanjing, China; ${ }^{3}$ Department of Pathology, Cordoba University, Cordoba, Spain; ${ }^{4}$ Institute of Pathological Anatomy and Histopathology, School of Medicine, Polytechnic University of the Marche Region (Ancona), United Hospitals, Ancona, Italy; ${ }^{5}$ Department of Pathology, Nanjing Medical University Affiliated Nanjing Hospital (Nanjing First Hospital), Nanjing, China and ${ }^{6}$ Department of Urology, Indiana University School of Medicine, Indianapolis, IN, USA

Glandular neoplasms involving the urinary bladder carry a challenging differential diagnosis including primary and secondary processes. We investigated the potential diagnostic utility of cadherin-17 and GATA3 in 25 primary adenocarcinomas of the urinary bladder, as compared with other commonly used markers including $\beta$-catenin and p63. Urothelial carcinoma with glandular differentiation (11), colorectal adenocarcinoma secondarily involving the bladder (25), and primary colorectal adenocarcinoma (22) were also analyzed and the results were compared using a Fisher exact test. Cadherin-17 was expressed in 23/25 primary bladder adenocarcinomas (92\%), 23/25 colorectal adenocarcinomas involving the bladder (92\%), 21/22 primary colorectal adenocarcinomas $(95 \%)$ and entirely negative $(0 / 11)$ in both components of urothelial carcinoma with glandular differentiation $(P<0.001)$. In urothelial carcinoma with glandular differentiation, positive nuclear staining for GATA3 was evident in the urothelial component for $18 \%(2 / 11)$ and the glandular component for $9 \%(1 / 11)$ with additional tumors showing only cytoplasmic staining. Nuclear reactivity for GATA3 was not present in primary bladder adenocarcinoma and primary/secondary colorectal adenocarcinoma $(P<0.05)$. Positive nuclear and cytoplasmic immunostaining for $\beta$-catenin was evident in $21 / 22$ primary colorectal adenocarcinomas (95\%) and $23 / 25$ cases of secondary involvement by colorectal adenocarcinoma $(92 \%)$. In contrast, positive membranous and cytoplasmic staining for $\beta$-catenin was observed in $23 / 25$ primary bladder adenocarcinomas $(92 \%)$ and $11 / 11$ urothelial carcinomas with glandular differentiation $(100 \%, P<0.001)$. p63 was expressed only in the urothelial component of urothelial carcinoma with glandular differentiation and not in the glandular component $(P<0.001)$. In summary, cadherin-17 is a relatively specific and sensitive marker for primary adenocarcinoma of the urinary bladder, distinguishing it from urothelial carcinoma with glandular differentiation. However, it does not distinguish primary bladder adenocarcinoma from secondary involvement by colorectal adenocarcinoma. The pattern of reactivity for $\beta$-catenin remains the most useful marker for distinguishing these two tumors. Modern Pathology (2013) 26, 725-732; doi:10.1038/modpathol.2012.229; published online 25 January 2013

Keywords: cadherin-17; $\beta$-catenin; GATA3; histologic variants; immunohistochemistry; urinary bladder; urothelial carcinoma with glandular differentiation

Correspondence: Dr L Cheng, MD, Department of Pathology, Indiana University School of Medicine, 350 West 11th Street, Indianapolis, IN 46202, USA.

E-mail: liang_cheng@yahoo.com

Received 26 September 2012; revised 9 November 2012; accepted 6 December 2012; published online 25 January 2013
Primary adenocarcinoma of the urinary bladder is an uncommon neoplasm, accounting for $0.5-2.0 \%$ of all malignant bladder tumors. ${ }^{1-3}$ Morphologically, these tumors show a wide spectrum of histologic appearances, including colonic (enteric), mucinous 
(so-called 'colloid'), signet ring cell, clear cell, mixed, and not otherwise specified subtypes., ${ }^{1,4-6}$ Therefore, distinguishing them from the secondary involvement of the urinary bladder by adenocarcinoma of another organ, especially the colon, is often challenging or impossible by light microscopy alone. ${ }^{6}$

Although immunohistochemical markers such as CK7, CK20, villin, CDX-2, and $\beta$-catenin have been proposed to aid in resolving the differential diagnosis of these tumors, limitations in sensitivity and specificity have prevented any single marker or small panel of markers from emerging as clearly superior. ${ }^{6-10}$ Differential diagnostic challenges remain and the identification of additional diagnostic tools is necessary. Cadherin-17 (CDH17), also called liver-intestinal cadherin or human peptide transporter-1, has been recently recognized as a sensitive and specific marker for adenocarcinoma of the digestive system, especially colorectal adenocarcinoma. ${ }^{11-15}$ Conversely, GATA3, a transcription factor, is a newly described marker of urothelial carcinoma. ${ }^{16-18}$ However, the expression of cadherin-17 and GATA3 in primary adenocarcinoma of the urinary bladder has not been well characterized. The aim of this study was to investigate the expression of cadherin-17 and GATA3 in primary adenocarcinoma of the urinary bladder and evaluate the potential diagnostic usefulness of these markers along with other commonly used markers such as $\beta$-catenin and p63.

\section{Materials and methods}

\section{Cases}

A total of 25 primary urinary bladder adenocarcinomas were analyzed including 19 colonic (enteric), three signet ring cell, and three mixed types. For comparison, 11 urothelial carcinomas with prominent glandular differentiation, 25 colorectal adenocarcinomas secondarily involving the urinary bladder, and 22 primary colorectal adenocarcinomas were analyzed. Clinical histories and hematoxylin and eosin (H\&E) slides of each case were reviewed to confirm the tumor origin.

\section{Immunohistochemistry}

Immunostaining was performed using the Dako Autostainer Plus (Dako, Carpinteria, CA, USA). The following antibodies were used: cadherin-17 (Novus, Littleton, CO, USA; 1:500 dilution), GATA3 (BioCare Medical, Concord, CA, USA; 1:50 dilution), p63 (Dako; 1:400 dilution), and $\beta$-catenin (Cell Marque, Rockland, CA, USA; prediluted/ready to use). Immunoreaction was performed using the EnVision Flex and Flex + Visualization Systems (Dako). Diaminobenzidine (3,3-diaminobenzidine) was used as the chromogen. Positive and negative controls stained appropriately.

The interpretation of immunoreactivity was performed in a semiquantitative manner by analyzing the extent of the positively staining tumor cells. The interpretation was performed as follows: 0 or negative, $0 \%$ tumor cell positivity; $1+$ or focal, $1-10 \%$ tumor cell positivity; $2+$ or moderate, $11-50 \%$ tumor cell positivity; and $3+$ or diffuse, $>50 \%$ tumor cell positivity. Results of immunohistochemical staining were analyzed with a two-tailed Fisher exact test. A $P$ value of $<0.05$ was considered statistically significant.

\section{Results}

Results of immunohistochemical staining are summarized in Table 1.

\section{Nonneoplastic Tissue Adjacent to the Tumor}

In nonneoplastic tissues, strong, diffuse membranous staining for cadherin-17 was detected exclusively in colorectal mucosa. GATA3 and p63 were exclusively expressed in nuclei of normal urothelium. Positive membranous staining for $\beta$-catenin was seen in both colorectal mucosa and urothelium.

\section{Primary Adenocarcinoma of the Urinary Bladder}

Cadherin-17 and $\beta$-catenin were each expressed in $92 \%(23 / 25)$ of tumors (Figure 1). The staining pattern was membranous for cadherin-17 and membranous and cytoplasmic for $\beta$-catenin, (Figures 1 and 2) although two tumors showed nuclear and cytoplasmic staining with antibody to $\beta$-catenin. There was a statistically significant difference in staining pattern (membranous and cytoplasmic vs nuclear) when compared with colorectal adenocarcinoma $(P<0.001)$. A variable percentage of tumor cells showed a positive reaction (cadherin-17: mean, 62\%; $\beta$-catenin: mean, 90\%). Staining for p63 was negative in all tumors. Unlike the nuclear expression of GATA3 in normal urothelium, primary urinary bladder adenocarcinoma showed only cytoplasmic reactivity, present in $60 \%$ of tumors (15/25). Differences in immunoreactivity between different subtypes of primary bladder adenocarcinoma were not observed.

\section{Urothelial Carcinoma with Glandular Differentiation}

None of the 11 cases of urothelial carcinoma with glandular differentiation was positive for cadherin17 in either the urothelial or glandular component, yielding no significant difference when compared with each other but a statistically significant difference when compared with adenocarcinomas 


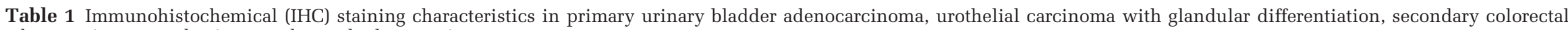
adenocarcinoma, and primary colorectal adenocarcinoma

\begin{tabular}{|c|c|c|c|c|c|c|c|c|c|c|c|c|c|c|c|c|c|c|c|c|c|c|c|c|c|}
\hline & \multicolumn{5}{|c|}{$\begin{array}{l}\text { Primary urinary bladder } \\
\text { adenocarcinoma }(\mathrm{n}=25)\end{array}$} & \multicolumn{10}{|c|}{ Urothelial carcinoma with glandular differentiation $(\mathrm{n}=11)$} & \multicolumn{5}{|c|}{$\begin{array}{l}\text { Secondary involvement of } \\
\text { bladder by colorectal } \\
\text { carcinoma }(\mathrm{n}=25)\end{array}$} & \multicolumn{5}{|c|}{$\begin{array}{l}\text { Primary colorectal } \\
\text { carcinoma }(\mathrm{n}=22)\end{array}$} \\
\hline & \multicolumn{5}{|c|}{ IHC scoring ${ }^{a}$} & \multicolumn{5}{|c|}{$\begin{array}{l}\text { Urothelial component } \\
\text { IHC scoring }\end{array}$} & \multicolumn{5}{|c|}{$\begin{array}{l}\text { Glandular component } \\
\text { IHC scoring }\end{array}$} & \multicolumn{5}{|c|}{ IHC scoring } & \multicolumn{5}{|c|}{ IHC scoring } \\
\hline & 0 & $1+$ & $2+$ & $3+$ & Total pos & 0 & $1+$ & $2+$ & $3+$ & Total pos & 0 & $1+$ & $2+$ & $3+$ & Total pos & 0 & $1+$ & $2+$ & $3+$ & Total pos & 0 & $1+$ & $2+$ & $3+$ & Total pos \\
\hline Cadherin-17 (membranous) & 2 & 2 & 5 & 16 & $23(92 \%)$ & 11 & 0 & 0 & 0 & 0 & 11 & 0 & 0 & 0 & 0 & 2 & 0 & 6 & 17 & $23(92 \%)$ & 1 & 0 & 2 & 19 & $21(95 \%)$ \\
\hline GATA3 (nuclear) & 25 & 0 & 0 & 0 & 0 & 9 & 0 & 0 & 2 & $2(18 \%)$ & 10 & 0 & 1 & 0 & $1(9 \%)$ & 25 & 0 & 0 & 0 & 0 & 22 & 0 & 0 & 0 & 0 \\
\hline p63 (nuclear) & 25 & 0 & 0 & 0 & 0 & 3 & 0 & 1 & 7 & $8(73 \%)$ & 11 & 0 & 0 & 0 & 0 & 25 & 0 & 0 & 0 & 0 & 22 & 0 & 0 & 0 & 0 \\
\hline$\beta$-Catenin (membranous) & 2 & 0 & 1 & 22 & $23(92 \%)$ & 0 & 0 & 0 & 11 & $11(100 \%)$ & 0 & 0 & 0 & 11 & $11(100 \%)$ & 23 & 0 & 0 & 2 & $2(8 \%)$ & 21 & 0 & 1 & 0 & $1(5 \%)$ \\
\hline$\beta$-Catenin (nuclear) & 23 & 0 & 0 & 2 & $2(8 \%)$ & 11 & 0 & 0 & 0 & 0 & 11 & 0 & 0 & 0 & 0 & 2 & 0 & 4 & 19 & $23(92 \%)$ & 1 & 0 & 0 & 21 & $21(95 \%)$ \\
\hline
\end{tabular}

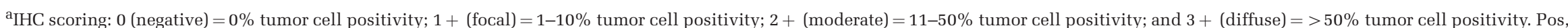

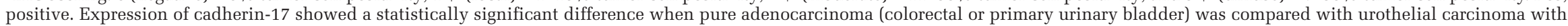

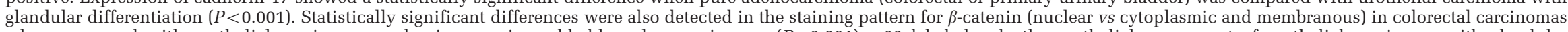
when compared with urothelial carcinomas and primary urinary bladder adenocarcinomas $(P<0.001)$. p63 labeled only the urothelial component of urothelial carcinoma with glandular differentiation $(P<0.001)$.
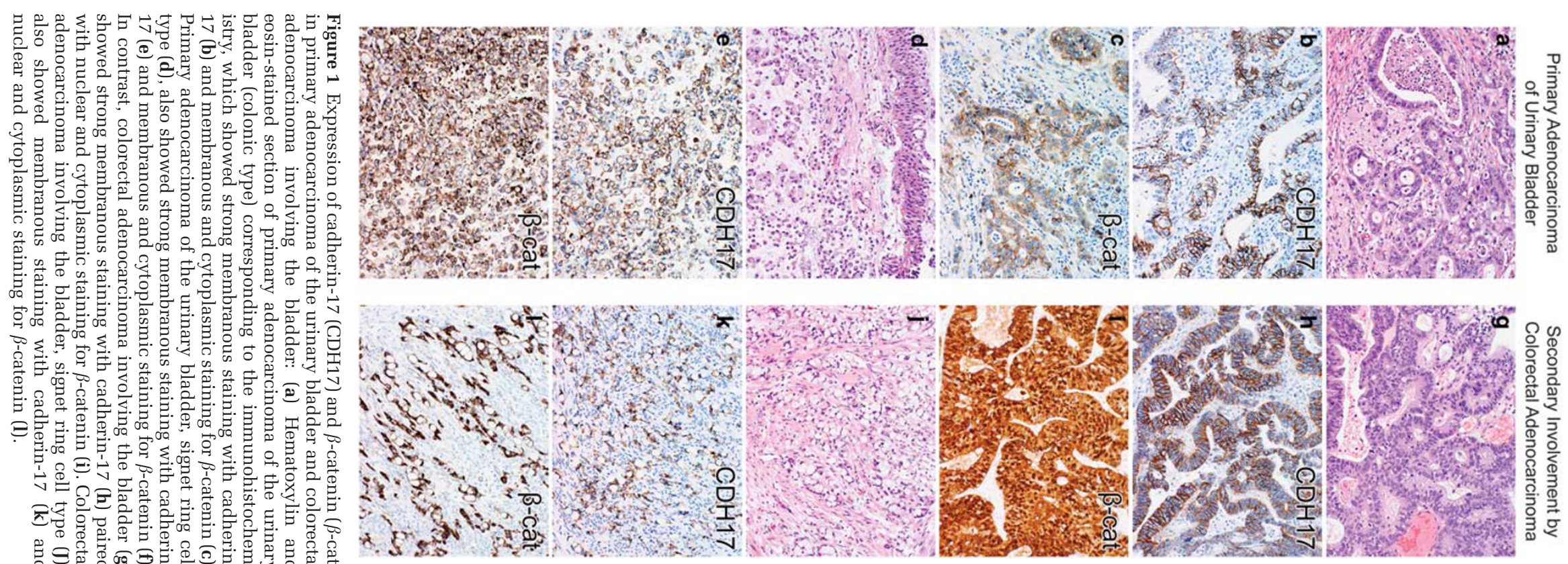

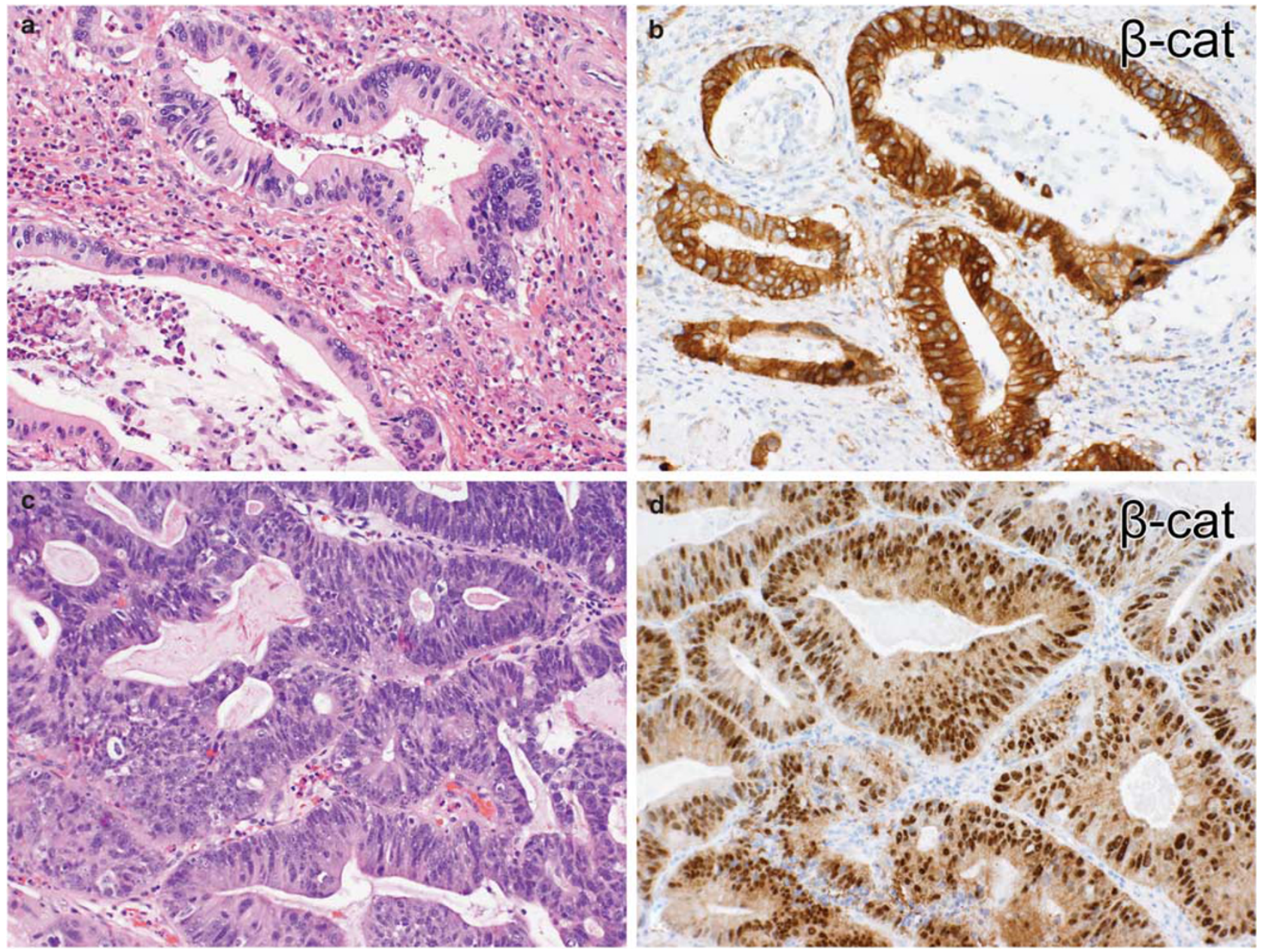

Figure 2 Expression patterns of $\beta$-catenin ( $\beta$-cat) in primary adenocarcinoma of the urinary bladder and colorectal adenocarcinoma involving the bladder: (a) Hematoxylin and eosin-stained section of primary adenocarcinoma of the urinary bladder (colonic type) corresponding to the immunohistochemistry. The tumor showed membranous and cytoplasmic staining with $\beta$-catenin (b). In contrast, colorectal adenocarcinoma involving the bladder (c) showed nuclear and cytoplasmic staining for $\beta$-catenin (d).

(colorectal and primary urinary bladder adenocarcinomas, $P<0.001)$. $\beta$-catenin showed membranous and cytoplasmic reactivity in both urothelial and glandular components of all 11 tumors (Figure 3). Therefore, no statistically significant difference was detected when comparing the two components to each other; however, a statistically significant difference was detected when compared with the nuclear staining pattern in colorectal adenocarcinomas $(P<0.001)$. Positive staining for p63 was present in the urothelial component for $73 \%(8 / 11$; nuclear staining), but negative in the glandular component of all cases $(P<0.001)$. Positive nuclear staining for GATA3 was evident in the urothelial component for $2 / 11$ tumors $(18 \%)$ and only a single tumor in the glandular component $(9 \%)$. No statistically significant difference was detected between the urothelial and glandular components; however, urothelial carcinomas showed increased staining when compared with pure adenocarcinomas (Table 1). Cytoplasmic staining for GATA3 was also present in some cases (36\%).

\section{Secondary Involvement by Colorectal Adenocarcinoma}

Cadherin-17 and $\beta$-catenin were each expressed in $92 \%$ of tumors (23/25) (Figures 1 and 2), resulting in no statistically significant difference when compared with primary urinary bladder adenocarcinoma $(P=1)$. Cadherin-17 labeled the tumor cells in a membranous pattern, while $\beta$-catenin showed nuclear and cytoplasmic staining. Some cases also showed concurrent membranous staining for $\beta$-catenin. Membranous reactivity without nuclear labeling for $\beta$-catenin was present in two tumors. A variable percentage of cells showed a positive reaction (cadherin-17: mean, 62\%; $\beta$-catenin: mean, $78 \%$ ). All cases were negative for p63 and nuclear expression of GATA3 was not present in any tumor, although cytoplasmic positivity for GATA3 was present in some tumors (48\%).

\section{Primary Colorectal Adenocarcinoma}

Membranous staining for cadherin-17 was observed in $95 \%(21 / 22)$ of primary colorectal adenocarcinomas 

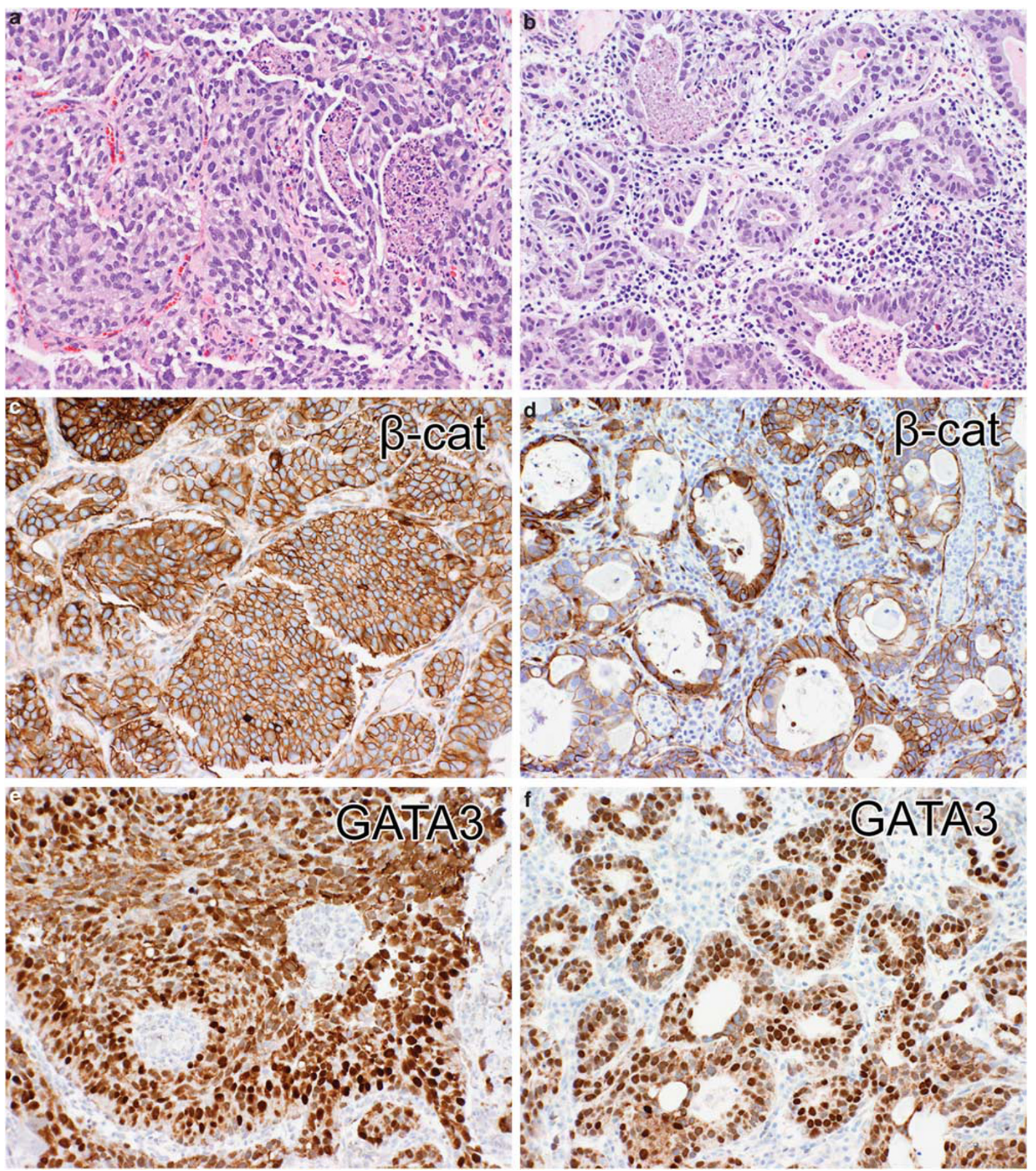

Figure 3 Expression of $\beta$-catenin ( $\beta$-cat) and GATA3 in urothelial carcinomas with prominent glandular differentiation: (a) Hematoxylin and eosin-stained section of the urothelial component of urothelial carcinoma with glandular differentiation. The urothelial carcinoma component showed membranous and cytoplasmic staining with $\beta$-catenin (b) and nuclear staining for GATA3 (c). The glandular component of this tumor (d) showed membranous and cytoplasmic staining with $\beta$-catenin (e) and nuclear staining for GATA3 (F), although nuclear expression of GATA3 was lacking in the majority of cases of urothelial carcinoma with glandular differentiation.

with a mean of $80 \%$ of tumor cells showing a positive reaction. None showed nuclear staining for GATA3 or p63, yielding no significant difference in p63 staining when compared with secondary adenocarcinoma or primary urinary bladder adenocarcinoma. Cytoplasmic labeling with GATA3 was frequently present $(86 \%)$. $\beta$-Catenin was expressed in the nuclei and cytoplasm of 21 primary colorectal 
adenocarcinomas with a mean of $97 \%$ of tumor cells showing a positive reaction. A single case showed only membranous and cytoplasmic staining for $\beta$-catenin.

\section{Discussion}

In this study, we have extended the known immunohistochemical profile of primary adenocarcinoma of the urinary bladder and confirmed some prior observations regarding these neoplasms. . $^{3,19,20}$ Cadherin-17, also called liverintestinal cadherin or human peptide transporter-1, is a sensitive and specific marker for adenocarcinomas of the digestive system, especially colorectal adenocarcinomas. ${ }^{11-15}$ In the study of $\mathrm{Su}$ et $a{ }^{12}$ expression of cadherin-17 was very common in colorectal adenocarcinomas (96\%) and evident in more than half of gastric and pancreatic adenocarcinomas. In contrast, cadherin-17 expression was very uncommon in carcinomas arising outside the gastrointestinal tract. Of 331 tumors including 44 breast cancers, 39 uterine cervical carcinomas, 50 lung cancers, 47 renal cell carcinomas, 89 ovarian cancers, and 39 prostatic cancers; cadherin-17 was expressed in only one $(0.3 \%)$ prostatic adenocarcinoma. ${ }^{12}$ These results support the utility of cadherin-17 as a useful diagnostic marker of gastrointestinal adenocarcinoma. Park et $a t^{21}$ similarly found a high concordance between the staining reactions in primary and metastatic colorectal carcinomas. In this study, we report that in addition to both primary and secondary colorectal adenocarcinoma, cadherin-17 is also commonly expressed in primary adenocarcinoma of the urinary bladder (92\%) and negative in urothelial carcinoma with glandular differentiation, a statistically significant difference $(P<0.001)$. Based upon these findings, cadherin-17 may be useful as a relatively specific and sensitive marker in the diagnosis of primary adenocarcinoma of the urinary bladder, distinguishing it from urothelial carcinoma with glandular differentiation. However, this marker does not distinguish primary adenocarcinoma of the urinary bladder from secondary involvement by colorectal adenocarcinoma.

Immunohistochemical antibody to transcription factor GATA3 has recently been recognized as a useful marker of urothelial differentiation. ${ }^{16-18}$ Unlike the usual nuclear expression pattern of GATA3 in normal urothelium and urothelial carcinoma, we identified only cytoplasmic reactivity in primary adenocarcinoma of the urinary bladder (60\%), colorectal adenocarcinoma $(86 \%)$, and secondary involvement of the urinary bladder by colorectal adenocarcinoma (48\%); nuclear expression was lacking. Previous studies have suggested a close histogenetic and pathogenetic relationship of primary adenocarcinoma of the urinary bladder and colorectal adenocarcinoma. ${ }^{22-26}$ Our finding that cadherin-17 and GATA3 expression are very similar in these two tumor types supports this relationship (Table 2). However, positive nuclear and cytoplasmic immunostaining for $\beta$-catenin was evident in $95 \%$ of $(21 /$ $22)$ primary and $92 \%$ of $(23 / 25)$ secondary colorectal adenocarcinoma, in contrast to positive membranous and cytoplasmic staining for $\beta$-catenin in $92 \%$ of $(23 / 25)$ primary adenocarcinomas of the urinary bladder and $100 \%$ of $(11 / 11)$ urothelial carcinomas with glandular differentiation $(P<0.001)$. These data indicate that dysregulation of $\beta$-catenin, an important aberration seen in colorectal carcinogenesis, does not appear to play a role in the pathogenesis of the bladder adenocarcinoma, ${ }^{27,28}$ confirming previous observations regarding the immunohistochemical profile of primary adenocarcinoma of the urinary bladder. ${ }^{3,7,19,20} \beta$-Catenin remains the most sensitive and specific marker for distinguishing primary adenocarcinoma of the urinary bladder from secondary involvement by colorectal adenocarcinoma.

We also evaluated this panel of biomarkers in 11 urothelial carcinomas with glandular differentiation. Of interest, p63 was expressed only in the urothelial carcinoma component (8/11 tumors) and not in the glandular component $(P<0.001)$. GATA3 showed expression only in a minority of cases (two in the urothelial component and one in the glandular component), a smaller percentage of

Table 2 General immunohistochemical features helpful in resolving the differential diagnosis of urinary bladder glandular malignancies

\begin{tabular}{|c|c|c|c|c|}
\hline & Cadherin-17 & GATA3 (nuclear) & $\beta$-Catenin & p63 \\
\hline $\begin{array}{l}\text { Primary urinary bladder } \\
\text { adenocarcinoma }\end{array}$ & Positive & Negative & Usually membranous & Negative \\
\hline $\begin{array}{l}\text { Urothelial carcinoma } \\
\text { with glandular differentiation }\end{array}$ & Negative & $\begin{array}{l}\text { Sometimes positive } \\
\text { (urothelial > glandular) }\end{array}$ & $\begin{array}{l}\text { Usually membranous for } \\
\text { both urothelial and } \\
\text { glandular } \\
\text { components }\end{array}$ & $\begin{array}{l}\text { Urothelial } \\
\text { carcinoma } \\
\text { component, usually } \\
\text { positive; glandular } \\
\text { component, } \\
\text { negative }\end{array}$ \\
\hline $\begin{array}{l}\text { Secondary involvement of bladder by } \\
\text { colorectal adenocarcinoma }\end{array}$ & Positive & Negative & Usually nuclear & Negative \\
\hline Primary colorectal adenocarcinoma & Positive & Negative & Usually nuclear & Negative \\
\hline
\end{tabular}


tumors than for urothelial carcinoma in general, which has been reported to be as high as $67-86 \%{ }^{16,18}$ Cadherin-17 was not expressed in either component of any tumor, contrasting to almost all primary adenocarcinomas of the urinary bladder, which were positive for cadherin-17 $(P<0.001)$. These prominent immunophenotypic differences between urothelial carcinoma with glandular differentiation and primary adenocarcinoma suggest that distinct mechanisms of histogenesis and pathogenesis are at play in the development of these tumors, despite histologic overlap in features.

In summary, in this study we have extended the immunohistochemical profile of primary adenocarcinoma of the urinary bladder (cadherin-17 positive, GATA3 negative, $\beta$-catenin membranous and cytoplasmic positive). Cadherin-17 is a relatively specific and sensitive marker in diagnosing primary adenocarcinoma of the urinary bladder, distinguishing it from urothelial carcinoma with glandular differentiation. However, it does not distinguish primary adenocarcinoma of the urinary bladder from secondary involvement by colorectal adenocarcinoma, in which case, $\beta$-catenin appears to remain the most useful marker. Although GATA3, when positive, supports a diagnosis of urothelial carcinoma with glandular differentiation, nuclear expression is only present in a minority of cases.

\section{Acknowledgements}

This study was supported by National Natural Science Foundation of China (81101933; Q Rao); Natural Science Foundation of Jiangsu Province, China (BK2010463; Q Rao) and Jiangsu Government Scholarship for Overseas Studies (W Huang). We thank Tracey Bender for her excellent editorial assistance.

\section{Disclosure/conflict of interest}

The authors declare no conflict of interest.

\section{References}

1 Cheng L, Lopez-Beltran A, Bostwick DG. Bladder Pathology. Wiley-Blackwell: Hoboken, NJ, 2012.

2 Grignon DJ, Ro JY, Ayala AG, et al. Primary adenocarcinoma of the urinary bladder. A clinicopathologic analysis of 72 cases. Cancer 1991;67:2165-2172.

3 Thomas AA, Stephenson AJ, Campbell SC, et al. Clinicopathologic features and utility of immunohistochemical markers in signet-ring cell adenocarcinoma of the bladder. Hum Pathol 2009;40:108-116.

4 Chor PJ, Gaum LD, Young RH. Clear cell adenocarcinoma of the urinary bladder: report of a case of probable mullerian origin. Mod Pathol 1993;6: $225-228$.
5 Roy S, Parwani AV. Adenocarcinoma of the urinary bladder. Arch Pathol Lab Med 2011;135:1601-1605.

6 Williamson SR, Lopez-Beltran A, Montironi R, et al. Glandular lesions of the urinary bladder: clinical significance and differential diagnosis. Histopathology 2011;58:811-834.

7 Wang HL, Lu DW, Yerian LM, et al. Immunohistochemical distinction between primary adenocarcinoma of the bladder and secondary colorectal adenocarcinoma. Am J Surg Pathol 2001;25:1380-1387.

8 Tamboli P, Mohsin SK, Hailemariam S, et al. Colonic adenocarcinoma metastatic to the urinary tract versus primary tumors of the urinary tract with glandular differentiation: a report of 7 cases and investigation using a limited immunohistochemical panel. Arch Pathol Lab Med 2002;126:1057-1063.

9 Suh N, Yang XJ, Tretiakova MS, et al. Value of CDX2, villin, and alpha-methylacyl coenzyme A racemase immunostains in the distinction between primary adenocarcinoma of the bladder and secondary colorectal adenocarcinoma. Mod Pathol 2005;18: 1217-1222.

10 Raspollini MR, Nesi G, Baroni G, et al. Immunohistochemistry in the differential diagnosis between primary and secondary intestinal adenocarcinoma of the urinary bladder. Appl Immunohistochem Mol Morphol 2005;13:358-362.

11 Ito R, Oue N, Yoshida K, et al. Clinicopathological significant and prognostic influence of cadherin-17 expression in gastric cancer. Virchows Arch 2005; 447:717-722.

$12 \mathrm{Su}$ MC, Yuan RH, Lin CY, et al. Cadherin-17 is a useful diagnostic marker for adenocarcinomas of the digestive system. Mod Pathol 2008;21:1379-1386.

13 Panarelli NC, Yantiss RK, Yeh MM, et al. Tissuespecific cadherin CDH17 is a useful marker of gastrointestinal adenocarcinomas with higher sensitivity than CDX2. Am J Clin Pathol 2012;138:211-222.

14 Ge J, Chen Z, Wu S, et al. A clinicopathological study on the expression of cadherin-17 and caudal-related homeobox transcription factor (CDX2) in human gastric carcinoma. Clin Oncol (R Coll Radiol) 2008; 20:275-283.

15 Lee HJ, Nam KT, Park HS, et al. Gene expression profiling of metaplastic lineages identifies CDH17 as a prognostic marker in early stage gastric cancer. Gastroenterology 2010;139:213-25 e3.

16 Higgins JP, Kaygusuz G, Wang L, et al. Placental S100 (S100P) and GATA3: markers for transitional epithelium and urothelial carcinoma discovered by complementary DNA microarray. Am J Surg Pathol 2007; 31:673-680.

17 Esheba GE, Longacre TA, Atkins KA, et al. Expression of the urothelial differentiation markers GATA3 and placental S100 (S100P) in female genital tract transitional cell proliferations. Am J Surg Pathol 2009;33:347-353.

18 Liu H, Shi J, Wilkerson ML, et al. Immunohistochemical evaluation of GATA3 expression in tumors and normal tissues: a useful immunomarker for breast and urothelial carcinomas. Am J Clin Pathol 2012;138: 57-64.

19 Gopalan A, Sharp DS, Fine SW, et al. Urachal carcinoma: a clinicopathologic analysis of 24 cases with outcome correlation. Am J Surg Pathol 2009;33:659-668.

20 Del Sordo R, Bellezza G, Colella R, et al. Primary signet-ring cell carcinoma of the urinary bladder: 
a clinicopathologic and immunohistochemical study of 5 cases. Appl Immunohistochem Mol Morphol 2009;17:18-22.

21 Park JH, Seol JA, Choi HJ, et al. Comparison of cadherin-17 expression between primary colorectal adenocarcinomas and their corresponding metastases: the possibility of a diagnostic marker for detecting the primary site of metastatic tumour. Histopathology 2011;58:315-318.

22 Bullock PS, Thoni DE, Murphy WM. The significance of colonic mucosa (intestinal metaplasia) involving the urinary tract. Cancer 1987;59:2086-2090.

23 Bates AW, Baithun SI. Secondary neoplasms of the bladder are histological mimics of nontransitional cell primary tumours: clinicopathological and histological features of 282 cases. Histopathology 2000;36:32-40.
24 Nakanishi K, Tominaga S, Kawai T, et al. Mucin histochemistry in primary adenocarcinoma of the urinary bladder (of urachal or vesicular origin) and metastatic adenocarcinoma originating in the colorectum. Pathol Int 2000;50:297-303.

25 Sung MT, Lopez-Beltran A, Eble JN, et al. Divergent pathway of intestinal metaplasia and cystitis glandularis of the urinary bladder. Mod Pathol 2006;19:1395-1401.

26 Morton MJ, Zhang S, Lopez-Beltran A, et al. Telomere shortening and chromosomal abnormalities in intestinal metaplasia of the urinary bladder. Clin Cancer Res 2007;13:6232-6236.

27 Chung DC. The genetic basis of colorectal cancer: insights into critical pathways of tumorigenesis. Gastroenterology 2000;119:854-865.

28 Fearon ER. Human cancer syndromes: clues to the origin and nature of cancer. Science 1997;278:1043-1050. 